\title{
ONLINE TUNING RULE BASED ADAPTIVE SPEED CONTROL ALGORITHM FOR DC MOTORS USING RECURSIVE LEAST SQUARES WITH FORGETTING
}

\author{
Kwang-seok Oh \\ Department of Mechanical Engineering \\ Hankyong National University \\ Anseong-si, 17579, Korea \\ oks@hknu.ac.kr
}

\author{
Jaho Seo \\ Department of Automotive, Mechanical and \\ Manufacturing Engineering \\ University of Ontario Institute of Technology \\ Oshawa, L1H 7K4, Canada \\ Jaho.Seo@uoit.ca
}

\begin{abstract}
This paper presents an online tuning rule-based adaptive control algorithm to control DC motor speed. For this, a method of recursive least squares with forgetting was proposed to approximate the relation between single input (voltage) and single output (angular speed) of the DC motor system as a first order differential equation. Using this approximated first order system and Lyapunov method-based disturbance observer with the online turning rule, a voltage input of the DC motor was generated to track a desired value of rotational speed of the motor. A performance evaluation of the proposed algorithm was conducted in the MATLAB/Simulink environment. The results show that the designed algorithm enables to track the reference speed successfully using only a single input and a single output of DC motor system.
\end{abstract}

Keywords- Lyapunov direct method; recursive least squares; single input single output; disturbance observer; DC motor system

\section{INTRODUCTION}

Modeling of dynamics in many systems including the DC motor is crucial for the purpose of dynamic analysis and control. However, the actual DC system has uncertainties, which cause a modeling error and thus have a negative effect on control performance. To deal with this issues, there are several existent studies to control the DC motor system that have been mainly based on model basis control approaches.

Ali et al. presented a model-based adaptive PID controller for the DC motor. The presented controller was able to secure robust and optimized control performance despite parameter variation and uncertainties [1]. Dursun and Durdu, proposed a speed control algorithm in the DC motor using a sliding mode observer that has been widely adopted in various industry control applications with uncertainties [2]. In addition, Meena and Bhushan suggested a DC motor speed control algorithm using fuzzy logic techniques [3]. In their study, a comparative analysis of the developed controller's performance with the PID control was conducted. The above studies require a mathematical model or knowledge of the systems to predict its dynamic behaviors and to derive an optimal input for successful control. However, it is hard to derive an accurate mathematical model (or obtain knowledge) for an optimal control in the actual system due to its nonlinearity and complexity. To address this issue, Xue et al. investigated a fractional order PID controller for a position servomechanism control system that dealt with actuator saturation and shaft torsional flexibility [4]. For an actual implementation, a modified approximation method was introduced in their study where some fixed parameters for the approximation were defined for the target system. However, the parameters need to be tuned whenever the target system is changed.

To overcome the above limitation, this study proposes a controller design method based on a model approximation that does not require parameters of the target system. In the method, an adaptive speed control algorithm with the online tuning was developed to control the DC motor speed using only single input (voltage) and single output (angular speed) of an approximated mathematical model (first order system). Also, this study introduces a disturbance observer using the Lyapunov direct method [5] that was designed to estimate disturbance.

The remainder of this paper is organized as follows. Section 2 provides a description of control concept. Section 3 describes the online tuning rule-based speed control algorithm. Section 4 provides a simulation-based performance evaluation results. Finally, Section 5 concludes the paper.

\section{CONCEPT OF CONTROL ALGORITHM}

A control algorithm proposed in this study was designed based on an approximated mathematical model that was assumed to have no restoring force due to frictional force existent in the actual system and thus can be finally considered as a damped first order system (Eq. (3)).

In the typical mechanical second order system, its dynamics can be expressed by the following equation.

$$
m \ddot{x}+c \dot{x}+k x+f=u
$$


where $m, c, k$ represent mass, damping coefficient, and stiffness, respectively. $x, u, f$ are the state variable, input and frictional term. If the stiffness $k$ can be assumed to have zero (no restoring force due to frictional force), the above equation can be arranged by Eq. (2) as follows.

$$
\ddot{x}+\frac{c}{m} \dot{x}+\frac{f}{m}=\frac{1}{m} u
$$

Since Eq. (2) does not include $k$ (restoring force) and thus it can be considered to be in a neutral equilibrium state. Then Eq. (2) can be rewritten as Eq. (3) by replacing $\dot{x}$ by $y$, which becomes a first order system.

$$
\dot{y}+\frac{c}{m} y+\frac{f}{m}=\frac{1}{m} u
$$

Since conditions of the first order damped system could be changed by various factors such as temperature, operating points, etc., the recursive least squares (RLS) was employed in this study to deal with an adaptive approximation of the damped system. Detailed explanation is provided in Section 3.

\section{ONLINE TUNING RULE BASED SPEED CONTROL ALGORITHM}

\section{A. RLS with forggeting based approximation}

For the adaptive approximation of damped system based on current input and output data, the RLS with forgetting method was applied. The first order differential equation used for this approximation is expressed in Eq. (4).

$$
\dot{y}+a y=u_{a}
$$

where $y$ is the system output that is controlled by the input $\left(u_{a}\right) . a$ is a time-varying parameter that represents the characteristic of the first order differential equation (4). To estimate the parameter $a$ adaptively using the system input and output, $u_{a}-\dot{y}, y$ and $a$ are defined as the output for the $\operatorname{RLS}\left(y_{r}\right)$, regressor $(\Phi)$, and estimate $(\hat{\theta})$, respectively. Fig. 2 shows a model schematic for the applied RLS with forgetting.

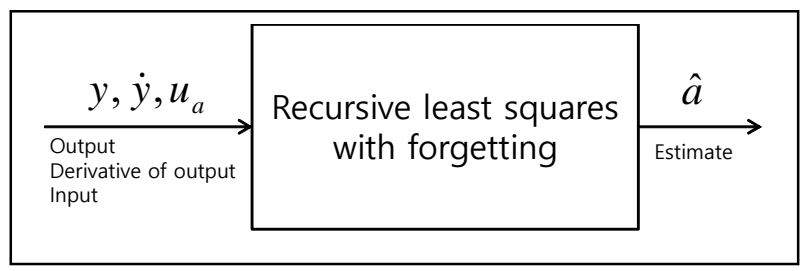

Figure 1. Model schematic for the RLS with forgetting
The following equations were used to compute the optimal gain $(L)$, and covariance $(P)$, and estimate $(\hat{\theta})$ that minimize the cost function $\left(J_{r}\right)$ with forgetting factor $(\lambda)$ in Eq. (8).

$$
\begin{gathered}
\hat{\theta}_{k}=\hat{\theta}_{k-1}+L_{k}\left(y_{r, k}-\Phi_{k} \hat{\theta}_{k-1}\right) \\
L_{k}=P_{k-1} \Phi_{k}\left(\lambda+\Phi_{k}^{T} P_{k-1} \Phi_{k}\right)^{-1} \\
P_{k}=\left(I-L_{k} \Phi_{k}^{T}\right) P_{k-1} / \lambda \\
J_{r}\left(\hat{\theta}_{k}, k\right)=\frac{1}{2} \sum_{i=1}^{k} \lambda^{k-i}\left(y_{r}(i)-\Phi(i) \hat{\theta}_{k}\right)^{2}
\end{gathered}
$$

\section{B. Disturbance observer}

The role of the Lyapunov method-based disturbance observer [5] adopted in the study is to estimate the disturbance that is a main source of modeling error between the approximately modeled damped system and actual one. The disturbance $(d)$ in the approximated first order equation is shown in the (9).

$$
\dot{y}=-\hat{a} y+u+d
$$

The used disturbance observer requires two stages of precorrection and estimation correction with the Lyapunov method. The following equation shows the disturbance observer's dynamics.

$$
\dot{e}_{y}=-\hat{a} e_{y}-e_{d}+K_{d}(y-\hat{y})
$$

where $e_{y}, e_{d}$, and $K_{d}$ represents the output error, disturbance error, and observer gain, respectively.

\section{Lyapunov direct method based control input}

The Lyapunov candidate function $(J)$ defined for control input is as follows.

$$
J=\frac{1}{2} e^{2}
$$

where $e$ represents the error $\left(e=y_{d e s}-y\right)$.

Using Eq. (12) below, a proper control input is derived so that the value of derivative of $J$ is always negative.

$$
\begin{aligned}
\frac{d J}{d t}=e \dot{e} & =\left(y_{d e s}-y\right)\left(\dot{y}_{d e s}-\dot{y}\right) \\
& =-W\left(y_{d e s}-y\right)^{2}
\end{aligned}
$$

where $W$ is the positive weighting factor that represents a change rate in the Lyapunov candidate function. Based on Eq. 
(9) and (12), The proper control input, $u_{p}$ that minimizes $J$ in Eq. (11) is derived as follows.

$$
u_{p}=\dot{y}_{d e s}+W y_{d e s}+(\hat{a}-W) y_{d e s}+\hat{d}
$$

The derived proper input can enable the output $(y)$ to track its desired output $\left(y_{d e s}\right)$. The derivative of the desired output $\left(\dot{y}_{d e s}\right)$ was estimated using a linear Kalman filter.

\section{Online tuning rule}

To cover the issue of degradation of control performance due to disturbance, the online tuning rule for $W$ with the error defined in Eq. (11) was utilized as shown in Eq. (14). While $W$ has a relatively large value in case of a large value of error, $\mathrm{W}$ has a relatively smaller value in the opposite case.

$$
W=m_{w} e^{2}+n_{w}
$$

where $m_{w}$ and $n_{w}$ are coefficients with negative values. Figure 2 shows the tuning rule in the $W$ and $e$ plane.

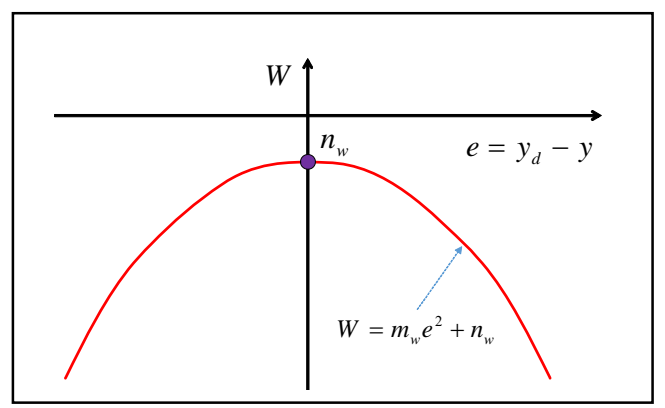

Figure 2. Model schematics for simulation model

\section{SIMULATION BASED PERFORMANCE EVALUATION}

Simulation was conducted to evaluate the designed controller's performance with a mathematical model of DC servo motor in the MATLAB/Simulink environment. Figure 3 shows a model schematic for the control performance evaluation.

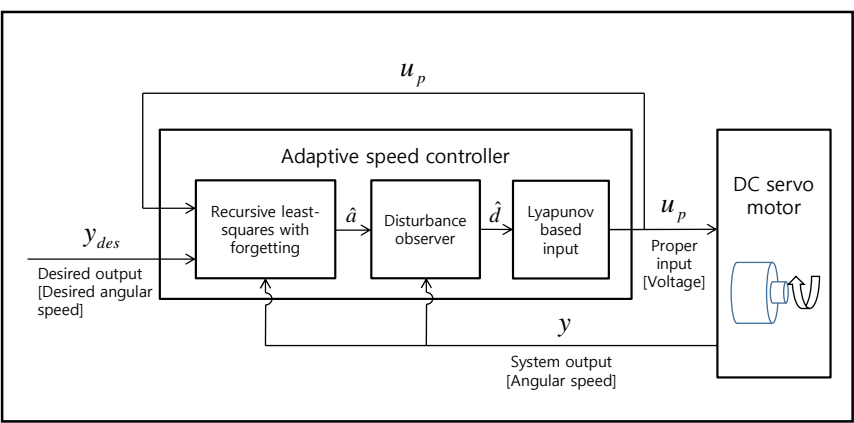

Figure 3. Model schematic for the control peformance evaluation
Figure 4 shows a typical circuit diagram of DC servo motor used in this study for the performance evaluation.

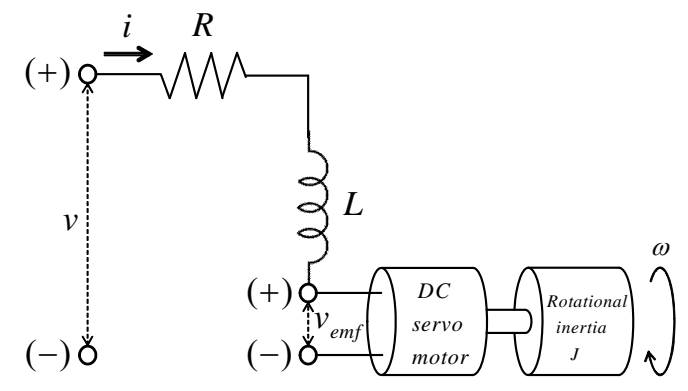

Figure 4. Circuit diagram of DC servo motor

In the figure, $R, L, \omega, i, v, v_{\text {emf }}, J$ represent the resistance, inductance, angular velocity, current, input voltage, counter electromotive force, and rotational inertia, respectively. Mathematical models of DC servo motor used for the performance evaluation are given in the following equations.

$$
\begin{gathered}
\frac{d i}{d t}=\frac{v}{L}-\frac{R}{L} i-\frac{K_{\Phi}}{L} \omega \\
\frac{d \omega}{d t}=\frac{K_{\Phi}}{J} i-\frac{b}{J} \omega
\end{gathered}
$$

where $K_{\Phi}$ represents the electromagnetic field constant. Based on the (15) and (16), the relationship between input (voltage, $v$ ) and output (angular speed, $\omega$ ) can be rewritten as follows.

$$
\dot{\omega}+\left(\frac{K_{\Phi}^{2}}{J R}+\frac{b}{J}\right) \omega+\left(\frac{K_{\Phi} L}{J R} \frac{d i}{d t}\right)=\left(\frac{K_{\Phi}}{J R}\right) v
$$

Using the RLS-based approximation method, the DC motor system model was approximated as shown in Fig. (1) and (3). Based on the approximated model and Lyapunov direct method in Eq. (13) and (14), a proper voltage input for DC motor speed control was derived. Table 1 shows parameter values used for the simulation-based evaluation.

TABLE I. THE PARAMETER VALUES FOR SIMULATION-BASED EVALUATION

\begin{tabular}{|c|c|c|}
\hline Division & Unit & Value \\
\hline Lambda $(\lambda)$ & - & 0.99 \\
\hline$R$ & $\Omega$ & 2 \\
\hline$L$ & $H$ & 0.1 \\
\hline$b$ & $N m s / r a d$ & 0.01 \\
\hline$J$ & $\mathrm{kgm}^{2}$ & 0.1 \\
\hline$K_{\Phi}$ & - & 0.3 \\
\hline$m_{w}$ & - & 0.7 \\
\hline
\end{tabular}




\begin{tabular}{|c|c|c|}
\hline Division & Unit & Value \\
\hline$n_{w}$ & - & -10 \\
\hline$W_{\text {constant }}$ & - & -10 \\
\hline
\end{tabular}

The performance of adaptive controller with the online tuning rule was compared with one with a constant weighting factor. Figs. 5-8 describes the evaluation results.

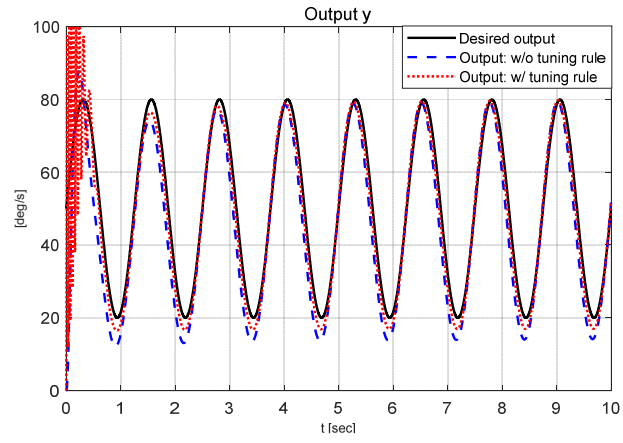

Figure 5. Evaluation results: output

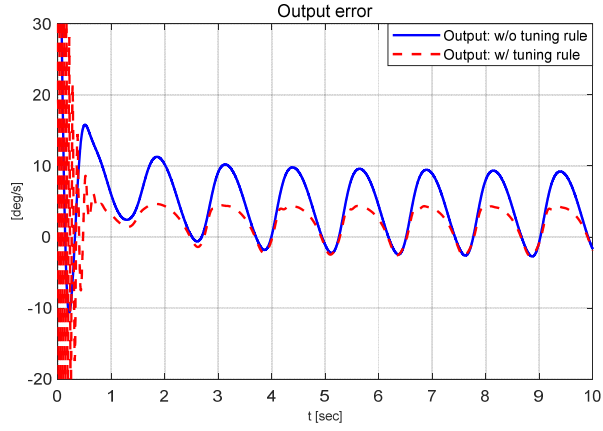

Figure 6. Evaluation results: output error

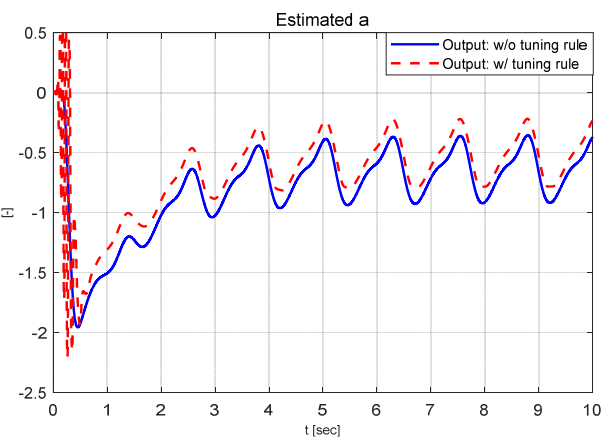

Figure 7. Evaluation results: estimated $a$

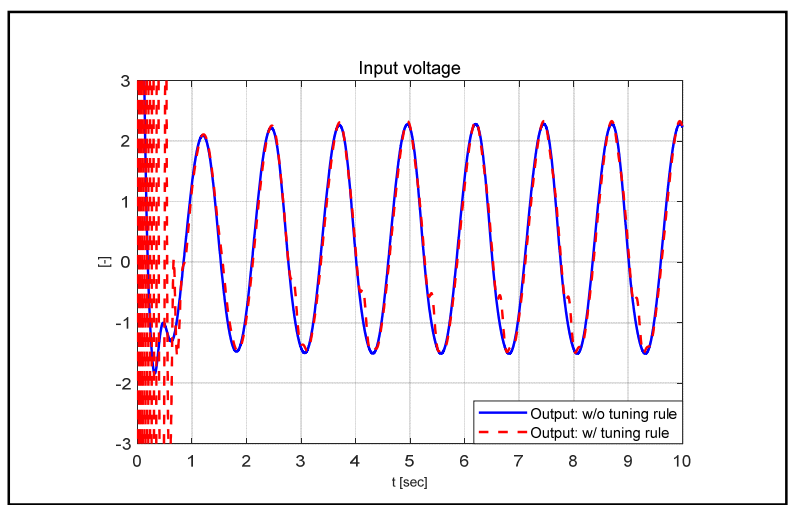

Figure 8. Evaluation results: voltage input

As seen in Figs. 5-6 and the Table 2 that shows the RMS values of output errors, the controller with the online tuning rule has smaller error than one with a constant weighting factor. However, the output $y$ in the online tuning rule-based control algorithm shows more oscillations compared to one with a constant weighting factor in the early stage of the simulation. Also, there are steady state output errors in both cases (Fig. 6). Oscillations in the estimated $a$ in both cases are found in Fig. 7, which are associated with oscillation of the desired output $y_{d}$. A shown in Fig. 8, there is no significant difference in the value of input voltage between both cases during the simulation time except for the beginning (i.e., transient state).

Table 2. RMS values of output errors

\begin{tabular}{|c|c|c|}
\hline Division & Unit & $\begin{array}{c}\text { RMS value } \\
\text { (Output error) }\end{array}$ \\
\hline $\begin{array}{c}\text { Without tuning rule } \\
\text { (With a constant weighting factor) }\end{array}$ & $\mathrm{deg} / \mathrm{s}$ & 7.69 \\
\hline With online tuning rule & $\mathrm{deg} / \mathrm{s}$ & 5.29 \\
\hline
\end{tabular}

\section{CONCLUSION}

In this study, the online tuning rule-based adaptive control algorithm was developed for controlling the DC motor system with single input (voltage) and single output (angular speed). For this, the approximated first order system and Lyapunov method-based disturbance observer with the online turning rule were utilized to design the control algorithms with the MATLAB/Simulink tool. The simulation results show that the system output with the designed controller accurately tracks the desired output. In addition, the RMS value of the output errors in the designed controller is smaller than the controller without tuning rule (i.e., constant weighting factor). However, both cases show oscillations at the beginning of simulation and steady state output errors. Therefore, a further study is required to improve the control performance to cope with this issue. It is expected that the proposed adaptive controller can be used as an alternative solution for the DC motor speed control in the related industrial applications.

\section{REFERENCES}


[1] A. Ali, E. Tayeb, and B. Mohd, "Adaptive PID controller for DC motor speed control," International Journal of Engineering Inventions, vol. 1, no. 5, pp. 26-30, 2012.

[2] E. Dursun and A. Durdu, "Speed control of a DC motors with variable load using sliding mode control," International Journal of Computer and Electrical Engineering, vol. 8, no. 3, pp. 219-226, 2016.

[3] P. Meena and B. Bhushan, "Simulation for position control of dc motor using fuzzy logic," International Journal of Electronics, Electrical and Computational System, vol. 6, no. 6, pp. 188-191, 2017.
[4] D. Xue, C. Zhao, and Y. Chen, "Fractional order PID control of a DCmotor with elastic shaft: A case study," Proceedings of the 2006 American Control Conference Minneapolis, Minnesota, USA, June 1416, pp. 3182-3187, 2006.

[5] C. Liu and H. Peng, "Disturbance observer based tracking control," Journal of Dynamic Systems, Measurement, and Control, vol. 122, pp. 332-335, 2000. 